\title{
ESTADO DE SALUD Y AUTOEFICACIA EN ADULTOS MAYORES USUARIOS DE ATENCIÓN PRIMARIA EN SALUD ${ }^{1}$
}

\section{HEALTH STATUS AND SELF -EFFICACY IN ELDERLY USERS IN PRIMARY CARE}

\author{
Catalina Tapia P. ${ }^{*}$ \\ VERÓNICA ITURRa M.** \\ YENNY VALDIVIA R.*** \\ Héctor VARELA V. \\ MÓNICA JORquera C. ${ }^{* * * * *}$ \\ Andrés Carmona G.
}

\begin{abstract}
RESUMEN
Objetivo: Identificar el nivel de autoeficacia percibida y su relación con variables biodemográficas y psicosociales en adultos mayores. Material y método: Diseño descriptivo correlacional, muestreo aleatorio estratificado, de 754 adultos mayores inscritos en centros de salud de Antofagasta, Chile. Mediciones: antecedentes sociodemográficos, estado de salud, autoeficacia percibida; Examen de Funcionalidad del Adulto Mayor (parte B); Calidad de vida relacionada con la salud, medida con el Cuestionario SF-12 y Fragilidad. Resultados: 61,4\% de mujeres versus 38,6\% de hombres; la edad osciló entre los 65 y 90 años, con una media de 73 años $(\mathrm{DE}=6,0)$; el 78,9\% declaró de dos a seis años de estudios. Los antecedentes mórbidos presentados con mayor frecuencia fueron: Hipertensión arterial, Dislipidemia y Diabetes Mellitus. El 86,3\% no presentó depresión. La autopercepción de salud calificada como "excelente a buena" alcanzó sobre el 60\%. La autoeficacia fue percibida alta en un $68,3 \%$. La calidad de vida relacionada con salud global fue percibida con satisfacción por el 55,6\%. Los niveles de fragilidad alcanzaron el 73,5\%. El 59,3\% de los adultos mayores fueron clasificados como autovalente sin riesgo. Se encontraron diferencias estadísticamente significativas entre las categorías de autoeficacia y temor a caerse, estado nutricional, depresión, calidad de vida relacionada con salud y la autopercepción de salud. Conclusión: Se identificó y relacionó una alta autoeficacia percibida, tanto en variables biodemográficas como psicosociales, que podría ser un factor facilitador para promover un envejecimiento activo.
\end{abstract}

Palabras clave: Estado de salud, autoeficacia, adultos mayores, atención primaria de salud, fragilidad.

\footnotetext{
${ }^{1}$ Financiado por Proyecto FONIS 2011, No SA11i2179.

* Enfermera. Dra. en Gerontología Social. Profesora Asociada Departamento de Enfermería Facultad Ciencias de la Salud. Universidad de Antofagasta, Chile. Email: catalina.tapia@uantof.cl. Autor de correspondencia.

** Enfermera. Magíster en Enfermería. Profesora Asistente Departamento de Enfermería Facultad Ciencias de la Salud. Universidad de Antofagasta, Chile. Email: veronica.iturra@uantof.cl

${ }^{* * *}$ Enfermera. Magíster en Enfermería. Profesora Asistente Departamento de Enfermería Facultad Ciencias de la Salud. Universidad de Antofagasta, Chile. Email: yenny.valdivia@uantof.cl

${ }^{* * * *}$ Dr. en Ciencias Matemáticas. Profesor Titular Departamento de Matemáticas. Facultad de Ciencias Básicas. Universidad de Antofagasta, Chile. Email: hector.varela@uantof.cl

${ }^{* * * * *}$ Enfermera. Magíster en Ciencias Sociales. Profesora Asistente Departamento de Enfermería Facultad Ciencias de la Salud. Universidad de Antofagasta, Chile. Email: monica.jorquera@uantof.cl

${ }^{* * * * * *}$ Enfermero. Magíster en Salud Pública. Profesor Asistente Departamento de Enfermería Facultad Ciencias de la Salud. Universidad de Antofagasta, Chile. Email: andres.carmona@uantof.cl
} 


\section{ABSTRACT}

Objective: Identify the level of perceived self-efficacy and its relationship with biodemographic and psychosocial variables in older adults. Material and method: Descriptive correlational design, stratified random sampling, of 754 older adults registered in health centers of Antofagasta, Chile. Measurements: sociodemographic background, health status, perceived self-efficacy; Functional Test of the Elderly (part B); Quality of life related to health, measured with the SF-12 and Fragility Questionnaire. Results: 61.4\% of participants were women and $38.6 \%$ were men; the ages ranged between 65 and 90 years, with an average of 73 years $(\mathrm{SD}=6.0) ; 78.9 \%$ declared between two and six years of schooling. The morbid antecedents most frequently presented were: Arterial Hypertension, Dyslipidemia and Diabetes Mellitus. 86.3\% did not present depression. Self-rated health rated "excellent to good" reached over $60 \%$. Self-efficacy was perceived as high by $68.3 \%$ of participants. A $55.6 \%$ was satisfied with their quality of life in relation to global health. Fragility levels reached $73.5 \% .59 .3 \%$ of older adults were classified as self-reliant without risk. Statistically significant differences were found between the categories of self-efficacy and fear of falling, nutritional status, depression, quality of life related to health and self-perception of health. Conclusion: A high perceived self-efficacy was identified and related, both in biodemographic and psychosocial variables, which could be a facilitating factor to promote active aging.

Key words: Health status, self-efficacy, elderly, primary health care, fragility.

Fecha recepción: 07/11/2016 Fecha aceptación: 27/12/2017

\section{INTRODUCCIÓN}

El foco del estudio del envejecimiento generalmente se centra más en aspectos biológicos como los deterioros y tratamientos, sin enfatizar en aspectos cualitativos como las conductas personales, que a lo largo de la vida determinan el tipo de envejecimiento y los mecanismos de adaptación a este proceso (1). En la Encuesta Nacional de Salud 20092010 (2) la población de 65 y más años presenta entre otros factores de riesgos cardiovasculares, sedentarismo $(96,1 \%)$, obesidad abdominal $(75,4 \%)$, hipertensión arterial $(74,6 \%)$ y síndrome metabólico $(51,6 \%)$. A nivel internacional la OMS declaró el 2009 que los factores de riesgo explican el 75\% de las enfermedades cardiovasculares (3), presentes desde la etapa de adulto joven, causante tanto de morbimortalidad como secuelas a nivel personal y familiar. Entre estos factores de riesgo asociados a las conductas personales se encuentran: el consumo excesivo de alimentos, alcohol, la escasa realización de ejercicios físicos, y el afrontamiento inadecuado del estrés (4); otra característica importante es que estos factores de riesgo se relacionan con los estilos de vida, que incorporan aspectos socioculturales, educacionales y económicos, sobre los cuales la persona puede ejercer diversos grados de control (5).

La diferencia entre la percepción de control (6) y la autoeficacia, es que el primer término involucra tanto factores externos como internos, mientras que la autoeficacia, según Bandura, se refiere principalmente a variables internas de la persona, entendida esta como: "las creencias de sus propias capacidades o los juicios que cada individuo tiene sobre sus capacidades para alcanzar con éxito un determinado logro o alcanzar un rendimiento deseado" (7).

La autoeficacia para los adultos mayores (AM) reviste una importancia tanto en sus capacidades físicas como en el sentimiento de poder participar activamente en la búsqueda de soluciones a conflictos cotidianos (8). Este sentimiento puede entenderse como empoderamiento que se asocia al sentido de autonomía, sensación de control y satisfacción que alberga el sentirse con poder (9). 
Diversas investigaciones han demostrado que altos niveles de autoeficacia se relacionan con efectos positivos para la salud y el bienestar de las personas $(10,11)$, además autoperciben mejor estado de salud y mejor pronóstico de recuperación de problemas de salud en general $(12,13)$. El estado de salud se evaluó en lo subjetivo a través de la autopercepción de salud y de temor a las caídas. En lo objetivo mediante los antecedentes mórbidos, estado nutricional, polifarmacia y caídas en el último año.

En esta investigación se planteó relacionar la autoeficacia como constructo de naturaleza psicosocial con variables biosociodemográficas, para enfatizar que la investigación centrada sólo en los aspectos biológicos del envejecimiento reduce la visión de éste y por tanto dificulta avanzar en la prevención del deterioro o en hallazgos que orienten políticas hacia un envejecimiento activo de la población.

Así el objetivo de este estudio fue identificar el nivel de autoeficacia percibida y su relación con: 1) variables biodemográficas; 2) variables psicosociales y 3 ) variables asociadas al estado de salud, que han demostrado relación teórica y/o empírica con la autoeficacia.

\section{MATERIAL Y MÉTODO}

Se utilizó un diseño descriptivo correlacional. El universo consideró la población de 65 y más años beneficiarios FONASA, e inscritos en algún centro de salud familiar de la ciudad de Antofagasta (CESFAM) que correspondió a 21.099 AM. La muestra fue aleatoria estratificada con afijación proporcional óptima y de selección simple a través de números aleatorios, considerando los listados de los AM de cada CESFAM. La información del tamaño de los estratos se obtuvo de la Corporación Municipal de Desarrollo Social de la ciudad. El tamaño de muestra calculado con 95\% de confianza y un margen de error en la estimación de la proporción de 0,04 corresponde a $632 \mathrm{AM}$, alcanzando una muestra de 754.

La recolección de los datos fue realizada en los CESFAM, mediante una entrevista personal, donde se aplicó la encuesta que consultaba sobre las siguientes variables:

a) Antecedentes sociodemográficos: edad, sexo, estado civil, nivel educacional, apoyo social y nivel de ingresos.

b) Estado de salud: antecedentes mórbidos (presencia de patologías); caídas en el último año y temor a éstas; estado nutricional; polifarmacia, según definición de la OMS (14); Depresión, medida a través de la Escala de Depresión Geriátrica de Yesavage (15) y Autopercepción de salud, medida a través de la consulta directa al AM (16).

c) Autoeficacia, medida a través de la Escala para determinar Autoeficacia percibida, validada para Chile (17).

d) Examen de funcionalidad del Adulto mayor (parte B del EFAM).

e) Calidad de vida relacionada con salud, medida con el Cuestionario SF-12, coeficiente alfa de Cronbach de 0,82 (18).

f) Fragilidad, medida en niveles según los indicadores de Fried (19): 1) pérdida de peso involuntaria superior a $4,5 \mathrm{k}$ del peso previo al último año, se comprobó con carnet de salud; 2) disminución en un $20 \%$ de la fuerza de agarre de la mano dominante, medido con un dinamómetro hidráulico (Jamer) ajustado según sexo e IMC; 3) pobre resistencia, definida por autorreporte de fatiga fácil frente a las actividades habituales; 4) actividad física, se aceptó válido el autorreporte de disminución de ésta. La ausencia de estos indicadores corresponde a la categoría sin fragilidad, la presencia de un indicador o más se consideró fragilidad $(20,21)$.

Los criterios de inclusión fueron tener 
edad mínima de 65 años y estar adscrito a algún centro de salud familiar (CESFAM). Los criterios de exclusión fueron: dependencia, discapacidad física, deterioro cognitivo severo, postrados, y/o analfabetos.

Previo a la recolección de datos se realizó una capacitación a las enfermeras de atención primaria y seminaristas de la carrera de Enfermería, de la Universidad de Antofagasta para estandarizar el manejo de las mediciones de la encuesta. La recolección de datos se realizó entre marzo y octubre de 2012.

Los requerimientos éticos que incluyen el consentimiento informado fueron sancionados y aprobados por el Comité de Ética en Investigación Científica de la Universidad de Antofagasta. En terreno, a los AM se les leía la información que indicaba los riesgos y beneficios de la investigación; según su respuesta se incorporaba o no al estudio.

Análisis de datos: Caracterización descriptiva de los resultados, con análisis de asociación, utilizando pruebas Chi cuadrado. El nivel de medición de las variables en su mayoría corresponde al nivel ordinal o nominal, por lo tanto se utilizó la prueba de Kruskal-Wallis para verificar si existen diferencias entre las categorías de autoeficacia. Los datos fueron procesados con el software SPSS, versión 20.

\section{RESULTADOS}

La muestra estuvo conformada en un $61,4 \%$ por mujeres; el rango de edad osciló entre los 65 y 90 años, con una media de 73 años (DE= 6,0 ). La distribución porcentual por categoría edad fue: $38,9 \%$ para los adultos mayores de 75 y más años; 32,9\% para los de 65-69 años y un $28,2 \%$ para los de 70 a 74 años. La categoría casado fue mayoritaria con un $40,8 \%$, seguido de viudo en un $37,3 \%$. En relación a la escolaridad, el 78,9\% declaró de dos a seis años de estudios. El ingreso económico con mayor representación alcanzó un 34,6\% para el rango de $\$ 80.000$ a $\$ 152.000$. El $58,6 \%$ se declaró sin apoyo social (Tabla 1).

Respecto del estado de salud: a) La autopercepción de salud, en su categoría buena, alcanzó la mayor distribución porcentual (48,5\%). b) Los antecedentes mórbidos de mayor frecuencia, fueron: Hipertensión arterial $(63,1 \%)$, Dislipidemia $(25,9 \%)$ y Diabetes Mellitus (24,4\%). Un 18,2\% presentaba Diabetes e Hipertensión arterial. El 26,5\% había presentado una caída en el último año y el temor a caerse duplicó ese valor $(56,8 \%)$. c) La alteración del estado nutricional se detectó como sobrepeso $(31,1 \%)$ y obesidad $(14,7 \%)$. d) Según la escala de Yesavage el $86,3 \%$ no presentaba depresión. e) La polifarmacia se encontró en un 35,9\% (Tabla 2).

La escala de autoeficacia, en esta muestra, alcanzó una confiabilidad de 0,836 según el coeficiente alfa de Cronbach, presentando un puntaje mínimo de 11 y máximo de 40 , con una media de 36,02 (DE 4,538), siendo percibida como alta en un $68,3 \%$ de los participantes. La calidad de vida relacionada con salud global se percibió con satisfacción en un $55,6 \%$ de la muestra. La fragilidad total fue el $73,5 \%$, un $69 \%$ en la categoría prefrágil y un $4,5 \%$ frágil. El examen de funcionalidad del AM (EFAM) clasificó como autovalente sin riesgo al 59,3\% de la muestra (Tabla 3).

A través de la prueba de Kruskal-Wallis se compararon los puntajes medianos de la escala de autoeficacia según las variables: edad categorizada, sexo, último curso de educación formal, estado civil y nivel de ingresos; en todas éstas la diferencia observada entre las medianas no fue estadísticamente significativa. Para las variables de salud se utilizó la misma prueba y se encontró que el temor a las caídas, el estado nutricional, la depresión, calidad de vida relacionada con salud y la autopercepción de salud presentaron una diferencia entre las dos medianas estadísticamente significativas; por tanto se puede plantear que la distribución de los puntajes en las categorías de autoeficacia es diferente para estas variables (Tabla 4). 
Tabla 1. Distribución porcentual de características biodemográficas en adultos mayores usuarios de atención primaria en salud.

\begin{tabular}{llrr}
\hline Variables & & $\mathrm{n}$ & $\%$ \\
\hline Sexo & Hombre & 291 & 38,6 \\
Categorías de edad (años) & Mujer & 463 & 61,4 \\
& $65-69$ & 248 & 32,9 \\
Estado civil & $70-74$ & 213 & 28,2 \\
& 75 y más & 293 & 38,9 \\
& Casado & 308 & 40,8 \\
& Viudo & 281 & 37,3 \\
& Soltero & 82 & 10,9 \\
& Divorciado & 71 & 9,4 \\
Último año escolaridad alcanzado & Solo lee y escribe & 12 & 1,6 \\
& Otros & 21 & 2,8 \\
Nivel de ingresos económicos & 595 & 78,9 \\
& 7 y más años & 138 & 18,3 \\
& $0-79.000$ & 72 & 9,5 \\
& $80.000-152.000$ & 261 & 34,6 \\
& $153.000-200.000$ & 178 & 23,6 \\
& 201.000-300.000 & 115 & 15,3 \\
Apoyo social & 301.000- 400.000 & 60 & 8 \\
& Mayor a 400.000 & 57 & 7,6 \\
& No contesta & 11 & 1,5 \\
& Sin apoyo social & 442 & 58,6 \\
& Apoyo social regular & 229 & 30,4 \\
& Apoyo social alto & 83 & 11 \\
\hline
\end{tabular}

1 dólar americano $=486$ pesos chilenos (2012).

Tabla 2. Distribución porcentual del estado de salud de adultos mayores usuarios de atención primaria en salud.

\begin{tabular}{llrc}
\hline Variables & & $\mathrm{n}$ & $\%$ \\
\hline Antecedentes mórbidos & Sin Patología & 126 & 16,7 \\
& Hipertensión arterial (HTA) & 476 & 63,1 \\
& Dislipidemia & 195 & 25,9 \\
& Diabetes Mellitus (DM) & 184 & 24,4 \\
& HTA y DM & 137 & 18,2 \\
& HTA, DM y Dislipidemia & 57 & 7,50 \\
& Artritis /Artrosis & 178 & 23,6 \\
Autopercepción de Salud & Mala & 28 & 3,70 \\
& Regular & 208 & 27,6 \\
& Buena & 366 & 48,5 \\
& Muy buena & 110 & 14,6 \\
& Excelente & 42 & 5,60 \\
\hline
\end{tabular}


Continuación Tabla 2.

\begin{tabular}{llrc}
\hline Caídas en el último año & Si & 200 & 26,5 \\
\multirow{3}{*}{ Temor a las caídas } & No & 554 & 73,5 \\
& Si & 428 & 56,8 \\
Estado Nutricional & No & 326 & 43,2 \\
& Enflaquecido & 94 & 12,5 \\
& Normal & 313 & 41,5 \\
Polifarmacia & Sobrepeso & 236 & 31,1 \\
& Obesidad & 111 & 14,7 \\
Depresión & Bajo consumo (0-2) & 483 & 64,1 \\
& Alto consumo ( 3 o más) & 271 & 35,9 \\
& Sin Depresión & 651 & 86,3 \\
& Depresión leve & 81 & 10,7 \\
& Depresión establecida & 22 & 3,00 \\
\hline
\end{tabular}

Tabla 3. Distribución porcentual de las escalas de autoeficacia, niveles de fragilidad y autovalencia según EFAM en adultos mayores usuarios de atención primaria en salud.

\begin{tabular}{llcc}
\hline Variables & & $\mathrm{n}$ & $\%$ \\
\hline Autoeficacia & Baja & 239 & 31,7 \\
& Alta & 515 & 68,3 \\
Calidad de vida & Satisfacción & 419 & 55,6 \\
& Insatisfacción & 335 & 44,4 \\
Niveles de fragilidad & Sin Fragilidad & 200 & 26,5 \\
& Prefrágil & 520 & 69,0 \\
& Frágil & 34 & 4,50 \\
& Autovalente con riesgo & 307 & 40,7 \\
Clasificación autovalencia según EFAM & Autovalente sin riesgo & 447 & 59,3 \\
\hline
\end{tabular}

Tabla 4. Prueba de Kruskal-Wallis. Autoeficacia y Estado de salud en adultos mayores, usuarios de atención primaria en salud.

\begin{tabular}{|c|c|c|c|c|c|}
\hline \multirow[b]{2}{*}{ Variables } & \multirow{2}{*}{$\begin{array}{l}\text { Categorías } \\
\text { Autoeficacia }\end{array}$} & \multirow[t]{2}{*}{$\mathrm{N}$} & \multirow{2}{*}{$\begin{array}{c}\text { Rango } \\
\text { promedio }\end{array}$} & \multicolumn{2}{|c|}{$\begin{array}{l}\text { Estadístico de } \\
\text { contraste }\end{array}$} \\
\hline & & & & $\mathrm{Chi}^{2}$ & valor $\mathrm{p}$ \\
\hline \multirow[t]{2}{*}{ Antecedentes Mórbidos } & Baja & 239 & 398,81 & 3,347 & 0,067 \\
\hline & Alta & 515 & 367,61 & & \\
\hline \multirow[t]{2}{*}{ Autovalencia (según EFAM) } & Baja & 239 & 355,91 & 4,755 & 0,029 \\
\hline & Alta & 515 & 387,52 & & \\
\hline \multirow[t]{2}{*}{ Temor a las Caídas } & Baja & 239 & 430,08 & 27,698 & 0,000 \\
\hline & Alta & 515 & 353,1 & & \\
\hline
\end{tabular}




\begin{tabular}{llllll} 
Continuación Tabla 4. & & & & \\
\hline Sufrió caídas el año anterior & Baja & 239 & 394,23 & 3,53 & 0,06 \\
& Alta & 515 & 369,74 & & \\
Estado Nutricional & Baja & 239 & 345,59 & 8,416 & 0,004 \\
& Alta & 515 & 392,31 & & \\
Polifarmacia & Baja & 239 & 391,51 & 0,653 & 0,067 \\
& Alta & 515 & 371,00 & & \\
Depresión & Baja & 239 & 418,94 & 35,664 & 0,000 \\
& Alta & 515 & 358,27 & & \\
Autopercepcion de Salud & Baja & 239 & 323,81 & 24,69 & 0,000 \\
& Alta & 515 & 402,42 & & \\
Calidad de vida relacionada con salud & Baja & 239 & 418,22 & 16,51 & 0,00 \\
& Alta & 515 & 358,60 & & \\
Fragilidad & Baja & 239 & 381,83 & 0,212 & 0,645 \\
& Alta & 515 & 375,49 & & \\
\hline
\end{tabular}

\section{DISCUSIÓN Y CONCLUSIÓN}

Los AM participantes en este estudio presentan características propias del envejecimiento a nivel nacional, son porcentualmente más mujeres, con edades sobre los 75 años; los ingresos económicos y la escolaridad se encuentran distribuidos mayoritariamente en las categorías bajas (22). Se declaró casado el $40,8 \%$, sin embargo el $58,6 \%$ percibe que no tiene apoyo social; este dato comparado con la encuesta de calidad de vida en la vejez 2013 (23) indica que la percepción de disponibilidad de apoyo varía de un 75,2 a $81,0 \%$ para diferentes situaciones, es decir la percepción de apoyo en esta muestra de AM antofagastinos fue baja; cabe considerar que en este estudio no se explicitaron situaciones en las que un AM requiere ayuda.

En relación a diversos aspectos de salud, los participantes en este estudio son autovalentes sin riesgo, según la clasificación del EFAM, en un 59,3\%, sin embargo presentan enfermedades crónicas como Hipertensión Arterial (63,1\%), Dislipidemia (25,9\%), Diabetes Mellitus $(24,4 \%)$, entre otras; además de la presencia de estas enfermedades, un $69,0 \%$ presenta al menos un indicador de fragilidad (24), síndrome geriátrico que aumenta con la edad y se correlaciona con un aumento de hospitalizaciones, complicaciones de enfermedades crónicas y degenerativas (25).

Destaca en esta muestra de AM, que un $86,3 \%$ resultó sin depresión, situación que puede ser interpretada como que lo orgánico no influyó en la esfera mental y/o emocional, lo que puede estar asociado con una calidad de vida en la categoría "satisfacción" 55,6\%, y percepción de salud en la categoría de "buena a excelente" en un $68,7 \%$, acompañado de una autoeficacia en la categoría alta en un $68,3 \%$. Estos resultados, para calidad de vida, autopercepción de salud y autoeficacia, en una muestra de AM con problemas o riesgos para su salud, tienen una característica esencial, son evaluaciones subjetivas, positivas que realiza el AM, que actúan como protectoras del estado de salud en general, desde el enfoque de la psicología positiva que estudia y promueve las potencialidades psíquicas de los individuos (13).

La percepción de autoeficacia y sexo, en este estudio no presentó diferencias significativas, hecho similar al estudio realizado en Buenos Aires (26) que no obstante encontró diferencias, éstas no tenían peso estadístico 
para ratificar esta significancia. Además, tanto en las diferencias de sexo como de edad, según plantea Schwarzer, uno de los autores de esta escala, pueden existir diferencias estadísticas, pero no implican una significancia psicológica (27). El nivel educacional no encontró diferencias significativas con la autoeficacia, contrario al estudio (28) en el cual ambas variables se correlacionaron con el nivel de compensación y la adherencia farmacológica en pacientes con Diabetes Mellitus.

En relación a las variables de salud, se encontró asociación de las variables: depresión, calidad de vida relacionada con salud, temor a las caídas, estado nutricional y autopercepción de salud con la autoeficacia general percibida; así se presentó una distribución de puntajes diferentes, con significancia estadística en todas estas variables; sin embargo, otras características de salud como son: antecedentes mórbidos, haber sufrido una caída, ser frágil o estar en riesgo no resultaron con variación en el puntaje de autoeficacia, lo que puede reflejar la naturaleza más psicosocial que psicobiológica del concepto; es decir una alta autoeficacia entrega al AM la sensación de bienestar psicológico sobre el estado de salud física que presentan (8).

Los participantes de este estudio en un $86,3 \%$ resultaron sin depresión, concordante con la alta autoeficacia $68,3 \%$; estos resultados son similares al estudio que encontró que una mayor autoeficacia se acompaña de menores síntomas depresivos en AM (29); esta relación confirma que altos niveles de autoeficacia se asocian con el bienestar general (10). Resultado similar se obtuvo con la calidad de vida global que en un $55,6 \%$ se percibió alta, sin embargo, existe un 44,4\% que no está satisfecho con la evaluación subjetiva y objetiva en las distintas dimensiones de su vida. Una limitación de este estudio es profundizar las distintas dimensiones de calidad de vida y la relación con la autoeficacia. La autopercepción de salud fue valorada por un $68,7 \%$ de "buena a excelente", mientras que en la encuesta de calidad de vida en la vejez, realizada en Chile en el año 2013, la misma categoría reporta un 38,9\% (23). Esta diferencia en los valores, es conceptualmente coherente con los altos puntajes de autoeficacia en esta muestra, al considerar que la autopercepción representa la percepción global que realiza una persona sobre su estado de salud y la autoeficacia son los juicios que una persona tiene sobre sus capacidades, ambos conceptos son predictores del estado de salud (30). El temor a las caídas (56,8\%) puede manifestar una pérdida de confianza en sí mismo para controlar y/o evitar la caída en un futuro (31), mientras que la autoeficacia es el juicio que realiza una persona en su presente, esta influencia temporal puede fundamentar que en esta muestra con alta autoeficacia, el temor a caerse sea alto. El IMC normal alcanzó la mayor distribución porcentual con un 41,5\%; sin embargo, si se suman las categorías de sobrepeso $(31,1 \%)$ y la obesidad $(14,7 \%)$, estas superan la categoría normal, dato concordante con la ENS 2009-2010 (2). Entre los factores psicológicos asociados a mantener un peso adecuado se ha estudiado la autoeficacia, la cual asociada a puntajes altos favorece la adherencia a diversos tratamientos para perder peso en adultos (32).

Esta investigación identificó la influencia positiva sobre algunos aspectos esenciales de salud y la alta autoeficacia, que facilita la cognición de las habilidades propias, que debe motivar a los profesionales de enfermería a realizar intervenciones a los AM para favorecer su percepción de autoeficacia, que estimula y mantiene la ejecución de conductas saludables que promueven un envejecimiento satisfactorio (33).

\section{REFERENCIAS}

1. González Bernal J, de la Fuente Anuncibay R. Desarrollo Humano. En: La vejez: Un envejecimiento óptimo desde los cuatro componentes del ser humano. Inter- 
national Journal of Developmental and Educational Psychology [Internet]. 2014 [citado 27 dic 2017]; 7(1): 121-129. Disponible: http://www.redalyc.org/articulo. oa? id=349851791013

2. Ministerio de Salud, Chile. Encuesta Nacional de Salud ENS Chile 2009-2010. Tomo II-V [Internet]. Santiago, Chile: MINSAL; 2010 [citado 04 abril 2016]. p. 2-282. Disponible en: http://web.minsal. $\mathrm{cl} /$ portal/url/item/bcb03d7bc28b64dfe040010165012d23.pdf

3. World Health Organization. Global health risks: Mortality and burden of disease attributable to selected major risks [Internet]. Geneva: World Health Organization; 2009. [citado 04 abril 2016]. 70 p. Disponible en: http://www.who.int/healthinfo/globalburdendisease/GlobalHealthRisks_report_full.pdf

4. Giraldo Osorio A, Toro Rosero MY, Macías Ladino AM, Valencia Garcés CA, Palacio Rodríguez S. La Promoción de la Salud como estrategia para el Fomento de Estilos de Vida Saludables. Hacia promoc. Salud [Internet]. 2010 [citado 10 jun 2016]; 15(1): 128-143. Disponible: http://www.scielo.org.co/pdf/hpsal/ v15n1/v15n1a09.pdf

5. López-Mateus MC, Hernández-Rincón $\mathrm{EH}$, Correal-Muñoz CA, Cadena-Buitrago GP, Galvis-Díaz IJ, Romero-Prieto GE. An educational strategy that promotes healthy habits in elderly people with hypertension in a municipality of Colombia: a participatory action research study. Medwave [Internet]. 2017 [citado 27 dic 2017]; 17(8): e7072. Disponible en: https://www.medwave.cl/link.cgi/ Medwave/Estudios/EstCualit/7072

6. Carpi Ballester A, Gonzales Navarro P, Zurriaga Llorens R, Marzo Campos JC, Buunk AP. Autoeficacia y percepción de control en la prevención de la enfermedad cardiovascular. Univ. Psych. 2010; 9(2): 423-432

7. Bandura A. Autoeficacia. En: Pensamien- to y acción: Fundamentos Sociales. Barcelona: Martínez Roca SA; 1987. p. 415478.

8. Ortiz J, Castro M. Bienestar psicológico de los adultos mayores, su relación con la autoestima y la autoeficacia. Contribución de enfermería. Cienc. enferm. 2009; XV(1): pp. 25-31.

9. Iacub R, Arias CJ. El empoderamiento en la vejez. Journal of Behavior, Health \& Social Issues [Internet]. 2010 [citado 16 jul 2016]; 2(2): 25-32. Disponible en: http://www.redalyc.org/articulo. oa? $\mathrm{id}=282221720003$

10. Reyes-Jarquín K, Hernández-Pozo MdR. Análisis Crítico de los estudios que exploran la Autoeficacia y Bienestar vinculados al Comportamiento Saludable. Journal of Behavior, Health \& Social Issues [Internet]. 2011 [citado 16 julio 2016]; 3(2): 5-24. Disponible en: http://www.journals.unam.mx/index.php/jbhsi/article/ view/29915

11. Sánchez JC. Efectos de la presentación del mensaje para realizar conductas saludables: el papel de la autoeficacia y de la motivación cognitiva. Int J Clin Health Psychol [Internet]. 2006 [citado 16 jul 2016]; 6(3): 613-630. Disponible en: http://www. redalyc.org/articulo.oa?id=33760307

12. Álvarez Ramírez LY, Ibarra L, García Espitia E. Eficacia de un programa para la promoción del bienestar a partir de las dimensiones del esquema de sí mismo en un grupo de adultos mayores de la ciudad de Bucaramanga. Psychol. av. discip [Internet]. 2011 [citado 27 dic 2017]; 5(1): 73-79. Disponible en: http://www.redalyc.org/articulo.oa?id=297224114007

13. Guerrero Alcedo JM, Requena Bastidas NY, Torrealba Torres ME. Bienestar psicológico, autoeficacia, estilos de humor y su relación con la salud. Revista Electrónica Medicina, Salud y Sociedad [Internet]. 2016 [citado 16 julio 2016]; 6 (2): 122-138. Disponible en: http://cienciasdelasaluduv.com/revistas/index.php/ 
mss/article/view/199

14. Santibáñez Beltrán S, Villarreal Ríos E, Galicia Rodríguez L, Martínez González L, Vargas Daza ER, Ramos López JM. Costo económico de la polifarmacia en el adulto mayor en el primer nivel de atención. Rev Med Inst Mex Seguro Soc [Internet]. 2013 [citado 27 dic 2017]; 51 (2): 192-199. Disponible en: dhttp://www. redalyc.org/pdf/4577/457745488015.pdf

15. Tapia C, Varela H, Barra L, Ubilla D, Iturra V, Collao C, et al. Valoración multidimensional del envejecimiento en la ciudad de Antofagasta. Rev Med. Chil. 2010; 138(4): 444-451.

16. Peláez E, Acosta LD, Carrizo ED. Factores asociados a la autopercepción de salud en adultos mayores. Rev Cubana Salud Pública [Internet]. 2015 [citado 28 mayo 2018]; 41(4): 638-648. Disponible en: http://www.redalyc.org/articulo. oa? id=21443545007

17. Cid P, Orellana A, Barriga O. Validación de la escala de autoeficacia general en Chile. Rev Med Chil. 2010; 138(5): 551557.

18. Urzúa A. Calidad de vida relacionada con la salud: Elementos conceptuales. Rev Med Chil. 2010; 138(3): 358-365.

19. Fried L, Tangen CM, Walston J, Newman AB, Hirsch C, Gottdiener J, et al. Frailty in older adults: evidence for a phenotype. J Gerontol A Biol Sci Med Sci [Internet]. 2001 [citado 16 jul 2016]; 56(3): 146-56. Disponible en: https://www.ncbi.nlm. nih.gov/pubmed/11253156

20. Rodríguez L, Castro M. Envejecimiento y fragilidad. En: Guillén Llera F, Molino Martín JP, Petidier Torregrosa R. Síndromes y cuidados en el paciente geriátrico. $2^{a}$ edición. Barcelona: Elsevier Masson; 2008. p. 21-30.

21. Abizanda P, Gómez-Pavón J, Martín I, Baztán J. Detección y prevención de la fragilidad: una nueva perspectiva de prevención de la dependencia en las personas mayores. Med Clín [Internet]. 2010 [citado 10 febr 2014]; 135(15): 713-719. Disponible en: http://www.elsevier. es/es-revista-medicina-clinica-2-articulo-deteccion-prevencion-fragilidad-una-nueva-S0025775309008422

22. Organización Mundial de la Salud. Informe Mundial sobre el Envejecimiento y la Salud [Internet]. Ginebra: Organización Mundial de la Salud; 2015 [citado 31 jul 2016]. 282 p. Disponible en: https://goo. gl/iAh8qY

23. Centro de Geriatría y Gerontología UC. Servicio Nacional del Adulto Mayor (SENAMA). Chile y sus mayores. Resultados Tercera Encuesta Nacional Calidad de Vida en la Vejez 2013 [Internet]. Santiago, Chile: UC; 2013 [citado 30 jul 2016]. 105 p. Disponible en https://goo.gl/gVzS$\mathrm{rg}$

24. Iriarte E, Araya AX. Criterios de fragilidad en personas mayores que viven en la comunidad: una actualización de la literatura. Rev Med. Chil. 2016; 144(11): 1440-47.

25. Molés Julio MP, Lavedán Santamaría A, Jürschik Giménez P, Nuin Orrio C, Botigué Satorra T, Maciá Soler L. Estudio de fragilidad en adultos mayores: diseño metodológico. Gerokomos [Internet]. 2016 [citado 27 dic 2017]; 27(1): 8-12. Disponible en: http://scielo.isciii. es/scielo.php?script $=$ sci_arttext $\&$ pi$\mathrm{d}=$ S1134928X2016000100003\&lg=es

26. Brenlla ME, Aranguren M, Rossaro MF, Vázquez N. Adaptación para Buenos Aires de la escala de autoeficacia general. Interdisciplinaria [Internet]. 2010 [citado 02 febr 2016]; 27(1): 77-94. Disponible en: https://goo.gl/2t6jJA

27. Schwarzer R, Born A. Optimistic self-beliefs: Assessment of general perceived self-efficacy in thirteen cultures. World Psychology. 1997; 3(1-2): 177-190.

28. Vergara Canales S, Barra Almagia E. Autoeficacia, apoyo social y adherencia al tratamiento en adultos con diabetes mellitus tipo II. Psicología y Salud [Internet]. 
2014 [citado 31 jul 2016]; 24(2): 167-173. Disponible en: https://goo.gl/VPXswR

29. Serra Taylor JA, Irizarry-Robles C. Factores Protectores de la Depresión en una Muestra de Adultos Mayores en Puerto Rico: Autoeficacia, Escolaridad y Otras Variables Socio-Demográficas. Act. colom psicol [Internet]. 2015 [10 ago 2016]; 18(1): 125-134. Disponible en: http://dx. doi.org/10.14718/ACP.2015.18.1.12

30. Zapata- Ossa H. Autopercepción de salud en adultos mayores y desenlaces en salud física, mental y síndromes geriátricos, en Santiago de Cali. Rev. salud pública [Internet]. 2015 [citado 11 ago 2016]; 17 (4): 589-602. Disponible en: http://bit. $\mathrm{ly} / 2 \mathrm{C} 9 \mathrm{sS} 1 \mathrm{~s}$

31. Curcio CL, Gómez Montes F. Temor a caer en ancianos: controversias en torno a un concepto y a su medición. Hacia promoc. Salud [Internet]. 2012 [citado
11 ago 2016]; 17(2): 187-204. Disponible en: http://www.scielo.org.co/pdf/hpsal/ v17n2/v17n2a13.pdf

32. Hernández Rodríguez S, Hernández Rodríguez R, Moreno Domínguez S. El papel de la autoeficacia percibida y la planificación en la adherencia al tratamiento médico-nutricional de la obesidad en adultos mexicanos. Revista Mexicana de Psicología. [Internet] 2015 [citado 11 ago 2016]; 32(1): 27-36. Disponible en: http://bit.ly/2DksrRn

33. Crespo-Sierra MT. Resiliencia, bienestar y aprendizaje a lo largo de la vida. International Journal of Developmental and Educational Psychology. Revista INFAD de Psicología [Internet]. 2016 [citado 27 dic 2017]; 1(2): 161-70. Disponible en: http://dx.doi.org/10.17060/ijodaep.2016. n2.v1.501 